\title{
MODELAGEM MATEMÁTICA E A DETERMINAÇÃO DE UM NOVO MÉTODO DE CÁLCULO DO VOLUME VENTRICULAR
}

\author{
José Sérgio Domingues ${ }^{1}$ \\ Kálita Gonçalves da Fonseca ${ }^{2}$ \\ Marcela Carvalho Gonçalves ${ }^{3}$ \\ Alex Eduardo Andrade Borges ${ }^{4}$
}

\begin{abstract}
RESUMO
Nesse trabalho, apresenta-se uma das aplicações da modelagem matemática no sistema cardiovascular, a sua utilização na estimação do volume ventricular esquerdo - um parâmetro fundamental no diagnóstico de doenças cardíacas. Para isso, prepara-se um molde de um ventrículo esquerdo bovino, seu volume real é determinado e, então, comparado com os resultados obtidos por métodos matemáticos médicos e com um novo método proposto, baseado na integral de revolução de uma função polinomial específica. Os resultados preliminares indicam que o método proposto é mais eficiente que os métodos médicos, com diferença de apenas $3,5 \%$ do volume real.
\end{abstract}

Palavras-chave: Modelagem Matemática. Novo Método de Cálculo. Volume Ventricular.

\section{MATHEMATICAL MODELING AND THE DETERMINATION OF A NEW METHOD OF THE VENTRICULAR VOLUME CALCULATION}

\begin{abstract}
In this work, we presented one of the applications of mathematical modeling to the cardiovascular system, its use in the estimation of the left ventricular volume, which is a basic parameter in the diagnosis of heart disease. To do that, a mold of a bovine left ventricle is prepared, its real volume is determined and then it is compared to the results achieved by medical mathematical methods and with a new proposed method, based on the integral of revolution of a specific polynomial function. Preliminary results indicate that the proposed method is more efficient than medical methods, with a difference of only $3.5 \%$ of the actual volume.
\end{abstract}

\footnotetext{
${ }^{1}$ Doutorando em Engenharia Mecânica/Bioengenharia pela Universidade Federal de Minas Gerais (UFMG). Mestre em Modelagem Matemática e Computacional pelo CEFET MG. Professor do curso de Matemática do IFMG - Campus Formiga. E-mail: sergio.domingues@ifmg.edu.br.

${ }^{2}$ Graduanda no curso de Licenciatura em Matemática do IFMG - Campus Formiga. E-mail: kalitagf@hotmail.com

${ }^{3}$ Graduanda no curso de Licenciatura em Matemática do IFMG - Campus Formiga. E-mail: marcelacg00@hotmail.com.

${ }^{4}$ Mestre em Matemática pela Universidade de São Paulo (USP). Professor do IFMG - Campus Formiga. E-mail: alex.borges@ifmg.edu.br.
} 
Keywords: Mathematical Modeling. New Calculation Method. Ventricular Volume.

\section{INTRODUÇÃO}

O volume ventricular esquerdo (VVE) está relacionado ao diagnóstico de várias doenças cardíacas, como hipertensão arterial, insuficiência cardíaca (IC) e uma das doenças mais severas para o coração, a Miocardiopatia Dilatada (MCD), caracterizada principalmente pela dilatação do ventrículo esquerdo, podendo levar o paciente ao quadro IC (GALRINHO et al., 2009; FERREIRA FILHO, 2012; MARTINS et al., 2008; DOMINGUES; BARBOSA; VALE, 2013).

Devido a essa importância, usa-se, nesse trabalho, um molde do ventrículo esquerdo bovino para analisar os modelos matemáticos destinados ao cálculo do VVE e comparar esses resultados com o resultado obtido em um novo Método Aqui Proposto (MAP). O coração bovino é usado devido à sua similaridade com corações humanos, distinguindo-se principalmente pelo tamanho e pela facilidade de obtenção. Espera-se que os resultados obtidos para eles possam ser também aplicados em humanos, o que motiva ainda mais esse trabalho.

O objetivo geral do projeto de iniciação científica registrado em nossa instituição é desenvolver modelos matemáticos de obtenção de VVE de bovinos com a utilização de métodos de interpolação e ajuste de pontos. O objetivo desse trabalho é apresentar essa análise para um molde, com um método de ajuste de pontos, e comparar os resultados com os métodos matemáticos médicos já utilizados em cardiologia.

\section{MATERIAIS E MÉTODOS}

As seguintes etapas foram necessárias: i) estudo do sistema cardiovascular, ii) estudo dos métodos de obtenção do molde ventricular, sua confecção, determinação das principais medidas e cálculo do seu volume real (VR), iii) análise dos principais métodos matemáticos médicos (Fig. 1), iv) obtenção do contorno ventricular, v) subdivisão do eixo das abscissas em partes iguais e obtenção dos pontos superiores determinados no contorno, vi) ajuste desses pontos por uma função polinomial de grau 6 e cálculo do volume do sólido de revolução gerado por essa curva. 


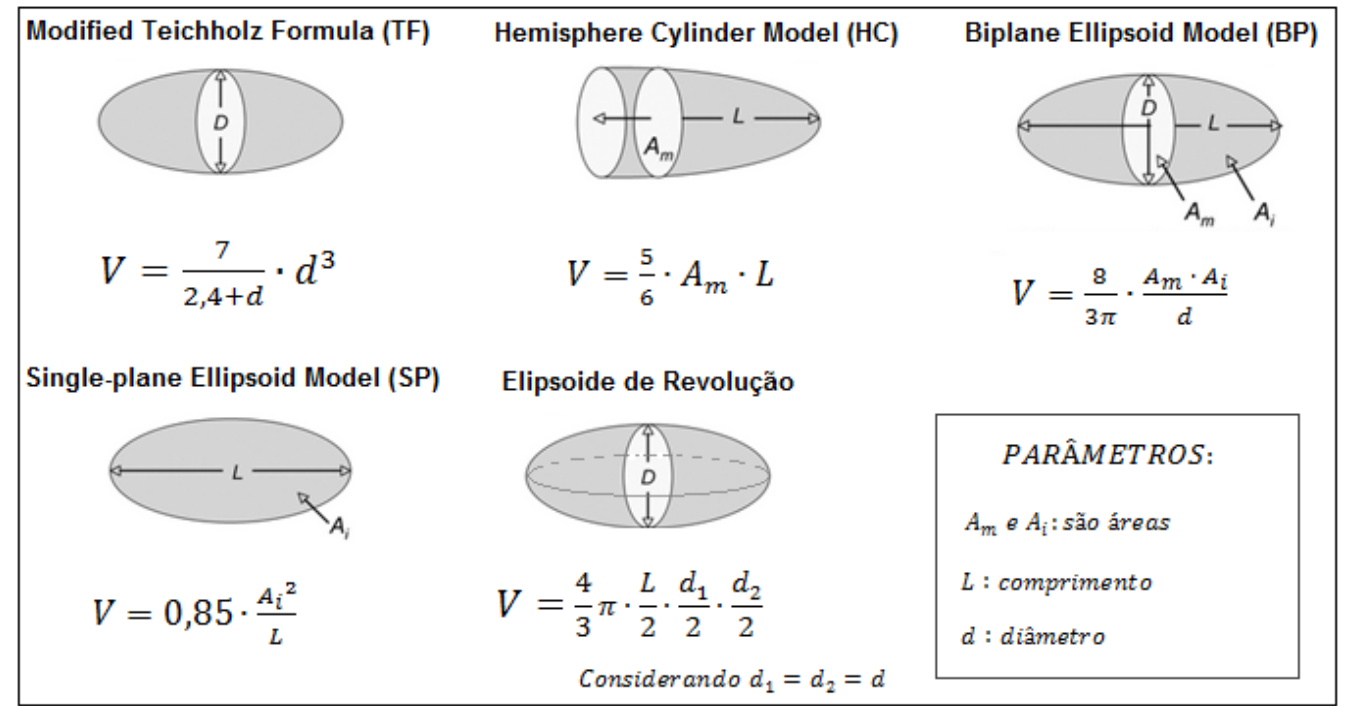

Figura 1 - Métodos Matemáticos Médicos

Fonte: Adaptado de Weijer et al., 2011.

Confeccionou-se o molde do ventrículo esquerdo de um bovino (Fig. 2), e seu VR, de valor 212,5 ml, foi calculado (pelo princípio de Arquimedes) seguindo os métodos descritos em Bezerra (1985). As medidas do molde foram obtidas utilizando-se um paquímetro universal, e através do corte do molde pelo plano transversal determinado pelos pontos médios aórtico e mitral e também pelo Apex, obteve-se o contorno ventricular, definido no plano cartesiano. A subdivisão do eixo das abscissas foi feita (29 partes iguais), e os pontos superiores do contorno foram determinados. Com esses dados, e utilizando o método dos mínimos quadrados em linguagem Matlab, foram calculados os coeficientes do polinômio de grau 6 que melhor se ajusta aos pontos obtidos (Eq. (1)).

$p(x)=0.00003 x^{6}+0.0005 x^{5}+0.0013 x^{4}-0.0225 x^{3}-0.1543 x^{2}+0.1296 x+3.3712$

Por fim, utiliza-se a Eq. (2) para calcular o volume do sólido de revolução gerado pelo polinômio $p(x)$ descrito na Eq. (1). Para isso, considera-se que $p(x)$ está definido no intervalo $[a, b]=[-8,8 ; 5,7]$, cujas extremidades representam o menor e o maior valor do eixo das abscissas utilizados na determinação do polinômio. O valor obtido foi $V=205,11 \mathrm{ml}$.

$$
V=\pi \int_{a}^{b}[p(x)]^{2} d x
$$


Todos os métodos matemáticos médicos descritos na Fig. 1 foram implementados em linguagem Matlab, e as medidas do molde confeccionado alimentaram suas equações. Os resultados obtidos para o VVE pelos métodos matemáticos médicos e pelo MAP foram comparados com o valor do VR.

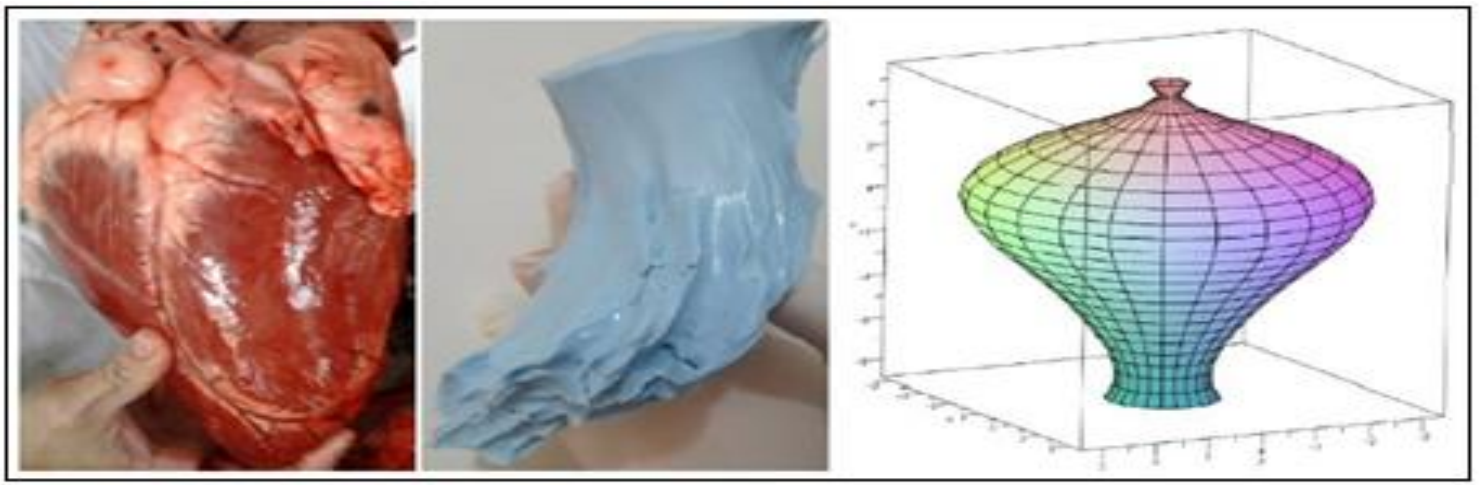

Figura 2 - Coração bovino, o molde confeccionado e o molde 3d feito em linguagem Maple®. Fonte: Elaborado pelos autores.

\section{RESULTADOS}

Para analisar os resultados obtidos através dos métodos matemáticos e do MAP, indicou-se o erro percentual referente a cada modelo, que podem ser visualizados na Tab. 1 .

Tabela 1 - Diferença percentual entre os métodos matemáticos e o volume real do molde.

\begin{tabular}{cc}
\hline Métodos matemáticos & $D=|(V R-V) / V R| \cdot 100$ \\
$V=\pi \int_{a}^{b}[p(x)]^{2} d x$ & $3,5 \%$ \\
$V=\frac{7}{2,4+d} \cdot d^{3}$ & $16,4 \%$ \\
$V=\frac{5}{6} A L$ & $12,9 \%$ \\
$V=\frac{8}{3 \pi} \cdot \frac{A_{m} A_{i}}{d}$ & $12,7 \%$ \\
$V=0,85 \cdot \frac{A_{i}{ }^{2}}{L}$ & $12,8 \%$ \\
$V=\frac{4}{3} \pi \frac{L}{2} \cdot \frac{D_{1}}{2} \cdot \frac{D_{2}}{2}$ &
\end{tabular}

Fonte: Elaborado pelos autores. 
No Graf. 1, é possível visualizar os volumes encontrados, utilizando os métodos médicos, o método aqui proposto e o volume real do molde.

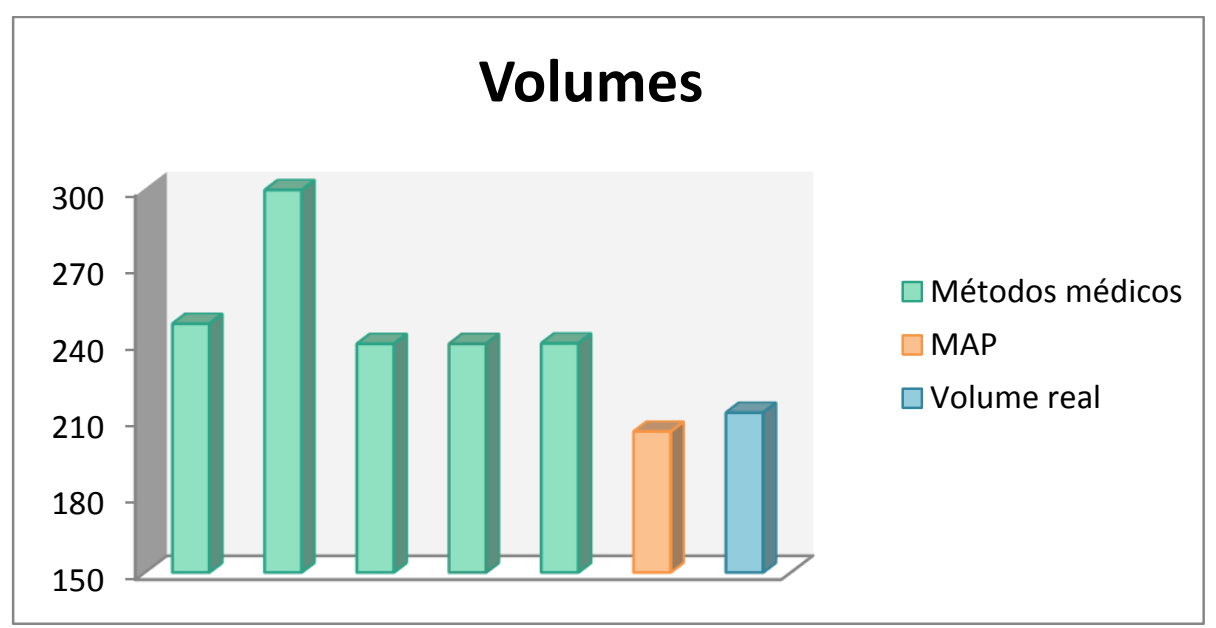

Gráfico 1- Comparação entre os diversos modelos matemáticos citados Fonte: Elaborado pelos autores.

O MAP apresentou maior concordância que os métodos matemáticos médicos, com diferença percentual de $3,5 \%$ em relação ao volume real, contra $12,7 \%$ de diferença nos melhores resultados obtidos pelos métodos médicos tradicionais.

\section{CONCLUSÃO}

Os resultados preliminares indicam boa concordância da maioria dos métodos matemáticos médicos em relação ao VR e uma concordância notável para o MAP.

O maior diferencial do MAP em relação aos métodos matemáticos médicos é que esses, em sua grande maioria, utilizam como referencial para o cálculo do VVE, formas geométricas pré-definidas (elipsoide). Com isso, algumas características relativas à geometria ventricular, específicas para cada indivíduo, não são consideradas, implicando em maior margem de erro. No MAP, uma vez que se toma como referência todo o contorno ventricular superior, essas características são bem consideradas, permitindo maior proximidade do valor obtido com o valor real calculado. Contudo, é claro que essa é uma primeira análise, relativa a apenas um molde, e que, portanto, precisa de maior fundamentação matemática e estatística para ser realmente validada. Sendo assim, visando ao aperfeiçoamento do método, novas análises serão realizadas e as etapas, que até o momento foram feitas manualmente, serão implementadas computacionalmente para que sua utilização possa ser feita de maneira semiautomática. 
AGRADECIMENTO: Os autores agradecem ao Programa Institucional de Bolsa de Iniciação Científica (PIBIC) do IFMG - Campus Formiga pelo apoio financeiro concedido.

\section{REFERÊNCIAS}

BEZERRA, S. J. Um modelo matemático para o cálculo do volume ventricular esquerdo. 1985. 73f. Dissertação (Mestrado em Matemática Aplicada) - Instituto de Matemática, Estatística e Ciência da Computação, UNICAMP, Campinas, 1985.

DOMINGUES, J. S.; BARBOSA, M. P.; VALE, M. de P. Mathematical Model for Partial Ventriculectomy. In: IEEE PORTUGUESE MEETING IN BIOENGINEERING (ENBENG)., $3^{\text {rd }}, 2013$, Braga. Proceedings... Braga: [s.n.], 2013. DOI 10.1109/ENBENG. 2013.6518422.

FERREIRA FILHO, P. R. P. F. Padrões de Hipertrofia e Geometria do Ventrículo Esquerdo pela Ecocardiografia Transtorácica. Rev bras ecocardiogr imagem cardiovasc, v. 25, n.2, p.103-115, 2012.

GALRINHO, A. et al. Volume da aurícula esquerda: um "velho" cálculo ecocardiográfico com uma importância prognóstica renovada. Um estudo em doentes com miocardiopatia dilatada. Rev Port Cardiol, v. 28, n.10, p.1049-1060, out.2009.

MARTINS, E. et al. Study Portuguese in dilated myocardiopathies Family. Rev Port Cardiol, v. 27, n.9, p. 1029-1042, set. 2008.

WEIJER, T. V. de et al. Geometrical models for cardiac MRI in rodents: comparison of qualification of left ventricular volumes and function by various geometrical models with a full-volume MRI data set in rodents. Am J Physiol Heart Circ Physiol. v. 302, p. H709 H715, nov. 2011. 